Check for updates

Cite this: J. Mater. Chem. B, 2021 9, 6988

Received 2nd April 2021 Accepted 12th May 2021 DOI: $10.1039 / \mathrm{d} 1 \mathrm{tb} 00730 \mathrm{k}$

rsc.li/materials-b

\section{Nanoparticle-mediated surfactant therapy in patients with severe COVID-19: a perspective}

\begin{abstract}
You $\mathrm{Wu}^{a}{ }^{\mathrm{a}}$ Xiaosi $\mathrm{Li}{ }^{\mathrm{a}}$ Yu Gan (D) ${ }^{\mathrm{b}}$ and Chao Zhao*a
Coronavirus disease 2019 (COVID-19) is an RNA virus-based disease that can be deadly. For critically ill patients, mechanical ventilation is an important life-saving treatment. However, mechanical ventilation shows a trade-off between supporting respiratory function and ventilator-induced lung injury (VILI). Surfactant therapy is a medical administration of exogenous surfactant to supplement or replace deficient or dysfunctional endogenous surfactant. Surfactant therapy can be used to postpone or shorten the use of mechanical ventilation to minimize or avoid VILI, because surfactants can reduce surface tension, improve lung compliance, and enhance oxygenation. In addition, nanotechnology can be applied to improve the therapeutic effect and reduce the adverse effects of surfactants. In this perspective, we discussed how nanoparticles deliver surfactants through intravenous injection and inhalation to the expected lung disease regions where surfactants are mostly needed, and discussed the prospects of nanoparticle-mediated surfactant therapy in the treatment of patients with severe COVID-19.
\end{abstract}

${ }^{a}$ Department of Chemical and Biological Engineering, The University of Alabama, P. O. Box 870203, Tuscaloosa, AL 35401, USA. E-mail: czhao15@eng.ua.edu; Tel: +01-(205)348-9869

${ }^{b}$ Department of Electrical and Computer Engineering, The University of Alabama, P. O. Box 870286, Tuscaloosa, AL 35401, USA

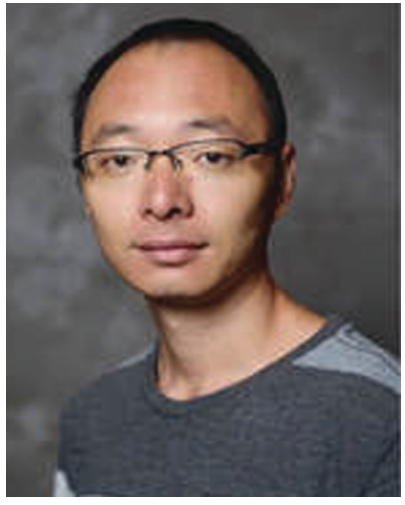

Chao Zhao
Dr Chao Zhao is an Assistant Professor in the Department of Chemical and Biological Engineering at the University of Alabama. He received his PhD degree in Chemical and Biomolecular Engineering from the University of Akron in 2013. He was a postdoctoral fellow at the University of Michigan during 2013-2016 and Harvard Medical School during 2016-2018. He joined the University of Alabama as an Assistant Professor as of July 2018. His research focuses on the polymer drug delivery systems in particular for small hydrophilic molecules and polymer scaffolds for tissue engineering. His work has been broadcasted in Boston 25 News and highlighted in 30+ national and international news agencies. At an early stage of his faculty career, he has been awarded the NIH Research Enhancement Award, University of Alabama RGC Level 1 Award, Alabama Transportation Institute grant, and U.S. Department of Education GAANN grant.

\section{COVID-19 and the lungs}

The outbreak of Coronavirus Disease 2019 (COVID-19) caused by the Severe Acute Respiratory Distress Syndrome Coronavirus 2 (SARS-CoV-2) has become a global health threat. In terms of the number of patients and the socioeconomic impact, COVID19 has greatly exceeded the other two diseases caused by coronaviruses, Severe Acute Respiratory Syndrome (SARS) and Middle East Respiratory Syndrome (MERS). COVID-19 generally affects the respiratory system, from airway compliance to epithelial integrity, and spans varying degrees of disease severity. At least $50 \%$ of people who test positive are asymptomatic or have mild illness. ${ }^{1}$ However, about $15-30 \%$ of patients develop lung complications, such as bilateral pneumonia., ${ }^{2,3}$ Among the patients presenting with COVID-19 pneumonia, $42 \%$ develop acute respiratory distress syndrome (ARDS), showing low lung compliance, shunting, and increased lung weight, with $61-81 \%$ of those requiring intensive care. ${ }^{4,5}$

The histopathological changes of COVID-19 patients mainly occur in the lungs. Immunohistochemical analysis confirmed the presence of SARS-Cov-2 antigen in the respiratory system, including the upper respiratory tract, bronchial epithelium, and alveolar epithelial cells. ${ }^{6}$ It is now known that initially, COVID-19 causes bilateral diffuse alveolar damage, followed by endothelial injury, pulmonary vascular endotheliitis, and alveolar-capillary injury, protein leakage, and alveolar wall inflammation. ${ }^{7}$ Noteworthily, COVID-19 patients who present severe hypoxemia can still present almost normal lung compliance, although other COVID-19 features may meet the ARDS definition. ${ }^{8}$ The rapid transition from almost-normal 
lung compliance to typical ARDS compliance may be due to the natural progression of pneumonia or ventilator-induced lung injury (VILI), which is an additional form of lung injury that generally occurs in ventilator-supported patients. ${ }^{9}$ Regardless of COVID-19 severity, mechanical ventilation in conjunction with positive end-expiratory pressure and the prone position has been shown to improve oxygenation in patients with hypoxemia. ${ }^{10,11}$

The alveolar epithelium produces and recycles endogenous pulmonary surfactant. Since COVID-19 damages the alveolar epithelium, ${ }^{12}$ the pulmonary surfactant should also be impaired by COVID-19. Hence, there is a strong biological plausibility of the use of surfactant therapy in COVID-19associated ARDS. A recent report shows that the amount of ventilatable lung tissue in an adult with severe ARDS is similar to that of a healthy 6-year-old child. ${ }^{13}$ Given that surfactant therapy has become a standard practice that is beneficial to neonate respiratory distress syndrome (RDS), ${ }^{14}$ surfactant therapy might be a candidate to treat severe COVID-19 patients who present hypoxemia but still have a non-stiff lung. The inclusion of surfactant therapy at the early stage of COVID-19 might postpone or avoid the use of a mechanical ventilator, thereby avoiding VILI.

\section{Exogenous surfactant replacement therapy}

The concept of exogenous surfactant therapy involves the delivery of exogenous surfactants, either animal-sourced or synthesized, to supplement or replace endogenous surfactant. In theory, lungs with dysfunctional or deficient endogenous surfactant should respond to the exogenous surfactant that spreads out as a monolayer at the air-liquid interface of the lungs. Such response is commonly accompanied by improved lung compliance, lowered ventilator plateau pressure, and/or improved gas exchange. In practice, it has been proven that exogenous surfactants can successfully improve the oxygenation and reduce the mortality of RDS newborns before 32 weeks of gestation, where surfactant deficiency has been believed to be the culprit. ${ }^{15}$ However, surfactant therapy has not been identified as a mortality benefit in adult ARDS patients, ${ }^{16}$ despite the fact that surfactant therapy trials reported short-term improvements in oxygenation and lung function. ${ }^{17}$ The negative outcomes of multiple surfactant therapy trials reduced the interest but provided opportunity for new research on the use of surfactant therapy to treat adult ARDS patients. In particular, the mechanism of surfactant therapy and its huge success in fighting against preterm RDS has inspired researchers to explore why surfactant therapy fails to reduce adult ARDS mortality.

The failure of surfactant therapy to reduce the mortality of adults with ARDS may be due to two reasons. First, for adults, the transport of surfactant from the trachea to the lungs is more challenging than for preterm infants. The lungs of preterm infants are commonly known to be edematous. The essentially edematous state dissolves the surfactant in water and the dissolved surfactant plays the role in reducing surface tension and improving lung compliance. Adult lungs are usually aerated, with the exception of a thin layer of liquid lining. Even in the case of illness, there are still aerated regions in adult lungs. This structural characteristic of the adult lung makes it difficult for the surfactant to dissolve in the water and subsequently reach the intended area of the lung. In addition, compared with neonatal lungs, surfactants need to travel through a longer airway branching system to reach the intended areas of the adult lungs. One commonly used method of surfactant administration is instillation. ${ }^{18}$ After being deposited in the trachea, exogenous surfactant will drain due to gravity through the first few airway generations. ${ }^{19}$ In the absence of additional driving force, it is challenging for the exogeneous surfactant to reach the lungs, due to the high viscosity of the surfactant complex and the airway cilia activities. $^{20,21}$ Thus, mechanical ventilation is usually used to drive the exogenous surfactant down to the alveoli. To overcome the high viscosity of surfactants, another commonly used administration of surfactant is through a nebulizer. But this method also has been characterized with low efficiency. ${ }^{22}$ Exogenous surfactant delivered by nebulization can reach unintended lung regions (e.g. an essentially air-filled, almostnormal region) that do not need the exogenous surfactants, leaving those edematous regions most in need of exogenous surfactant untreated. Traditional administration of surfactant also has been found to potentially cause overwhelm of the cardiopulmonary system, which could also obscure its benefits. ${ }^{23}$ Second, even if the surfactant is delivered to the intended disease area, the exogenous surfactant loses its surface-active function. During the journey to the alveoli, the airway contents exhibit a wide range of content compositions, and physical and chemical properties, and varying levels of abnormality in diseased lungs, and these contents may change the surface-active properties of exogenous surfactants. Depending on the administration method and lung disease conditions, exogenous surfactants maintain different levels of surface-active function. ${ }^{24,25}$

Therefore, the administration and delivery of exogenous surfactants to the intended lung regions with intact or wellpreserved surface-active properties plays a key role in replacing the endogenous surfactant and function as anticipated. To date, more efficient delivery of intact exogenous surfactants with maximal surface-active properties is in need. Nanoparticles hold potential in resolving these issues by delivering surfactant complexes to the lung regions that need surfactant most and eventually lowering VILI and mortality.

\section{Prospects of nanoparticle-mediated surfactant therapy for patients with COVID-19 ARDS}

Materials science and nanotechnology have been widely explored as weapons against the COVID-19 pandemic in many aspects, such as personal protective equipment supplies, ${ }^{26}$ 
medical equipment and instruments,${ }^{27}$ rapid detection and diagnosis platforms,${ }^{28}$ and vaccine formulations. ${ }^{29}$ In addition to these applications, although there are no clinically recognized antiviral treatment options, materials science and nanotechnology are also expected to be used for drug delivery to treat COVID-19. ${ }^{30,31}$

The use of nanoparticles as a carrier is a promising approach to deliver surfactants to the lungs to enhance the therapeutic efficacy of the surfactants and minimize undesirable side effects. Some of the main advantages of using nanoparticles to deliver surfactants include increasing resistance time in the body (increasing half-life of clearance) and targeting of surfactant to specific locations in the lung. There are many different kinds of known nanoparticles, such as polymer-based nanoparticles, lipid-based nanoparticles, inorganic nanoparticles, drug conjugates, and viral nanoparticles, etc. ${ }^{32,33}$ (Fig. 1). Surfactants can be physically encapsulated in these nanoparticles or covalently conjugated to the nanoparticles.

The surfactant-loaded nanoparticles can be injected intravenously. For example, surfactants can be loaded into polyethylene glycol (PEG) decorated liposomes and/or poly(lacticco-glycolic acid) (PLGA) nanoparticles. After intravenous injection, the PEG decorated nanoparticles can have a longer blood circulation to prevent the administered surfactant from being quickly cleared by macrophages. In addition, due to the enhanced permeability and retention (EPR) effect in inflammatory tissues, nanoparticles can be locally enriched at the site of pulmonary insult in the lungs to achieve targeted delivery of the surfactant ${ }^{34}$ (Fig. 2). Therefore, nanoparticles can increase the efficacy of surfactants while reducing their side effects.

The surfactant-loaded nanoparticles can also be administered by inhalation. One challenge of inhaling surfactants is that most of the surfactants are delivered to the airways or the lung periphery rather than the specific lung injury regions. To address this issue, the surfactant can be loaded in magnetic aerosol droplets reported by Dames and colleagues. ${ }^{35}$ The aerosol droplets contain superparamagnetic iron oxide nanoparticles (SPIONs). After inhalation, aerosol droplets can be accumulated in a specific lung region by a magnetic field, thereby achieving targeted delivery of surfactant to the murine lung injury area, where surfactants can be released from the nanoparticles (Fig. 3). ${ }^{36}$

In the nanoparticle-enveloped environment, the exogenous surfactant would be guided towards and target the lung regions that need exogenous surfactant the most. During the journey to edematous lung regions, enveloped and protected by the nanoparticles, the surfactant complex would have minimal interaction with the airway content until they reach the alveolar space. Without the airway content interference, the surfaceactive property of the exogenous surfactant is presumably

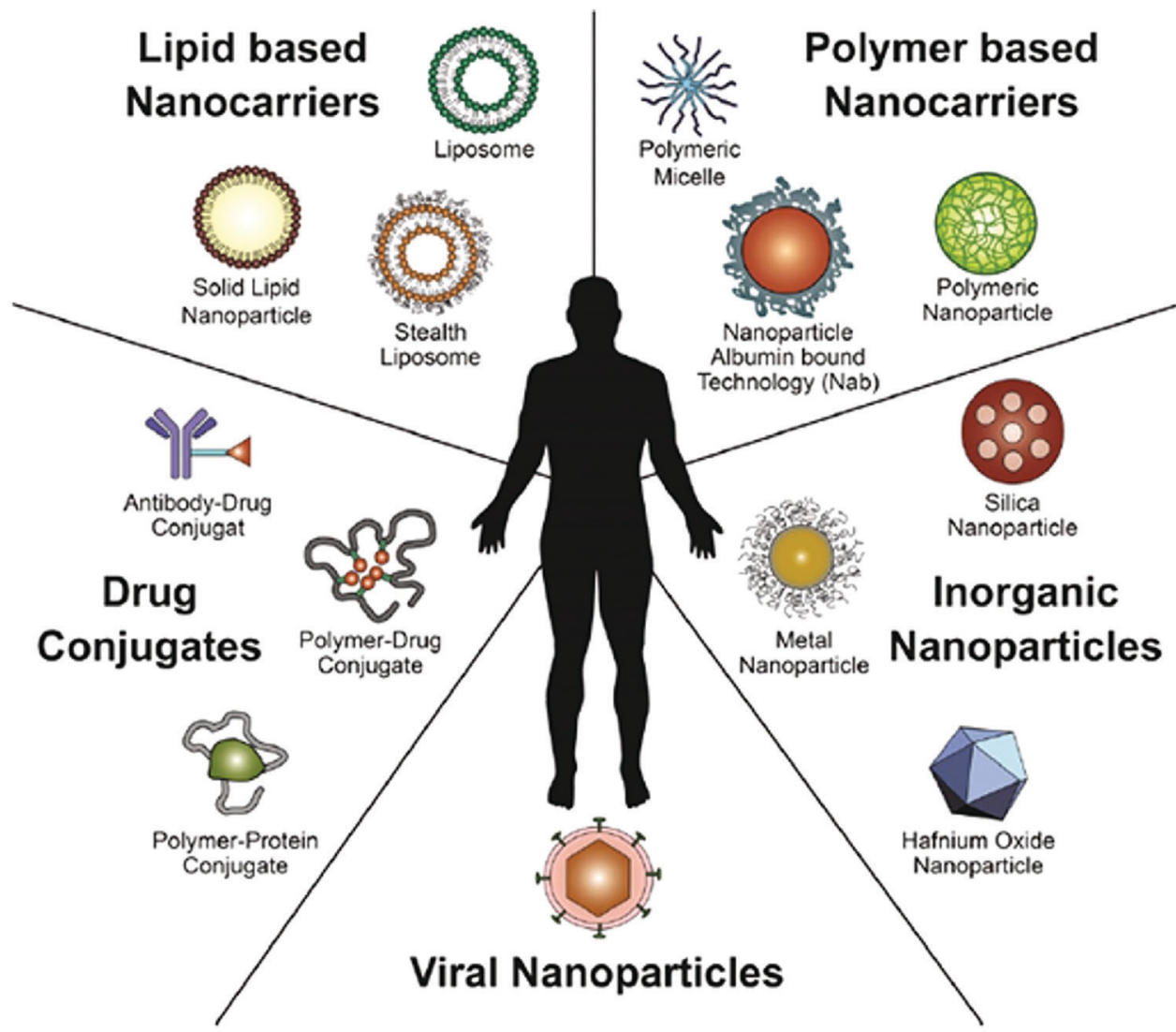

Fig. 1 Examples of nanoparticles commonly used as drug delivery carriers (reprinted with permission from Journal of Controlled Release, 2015, 200, $\left.138-157^{32}\right)$. 


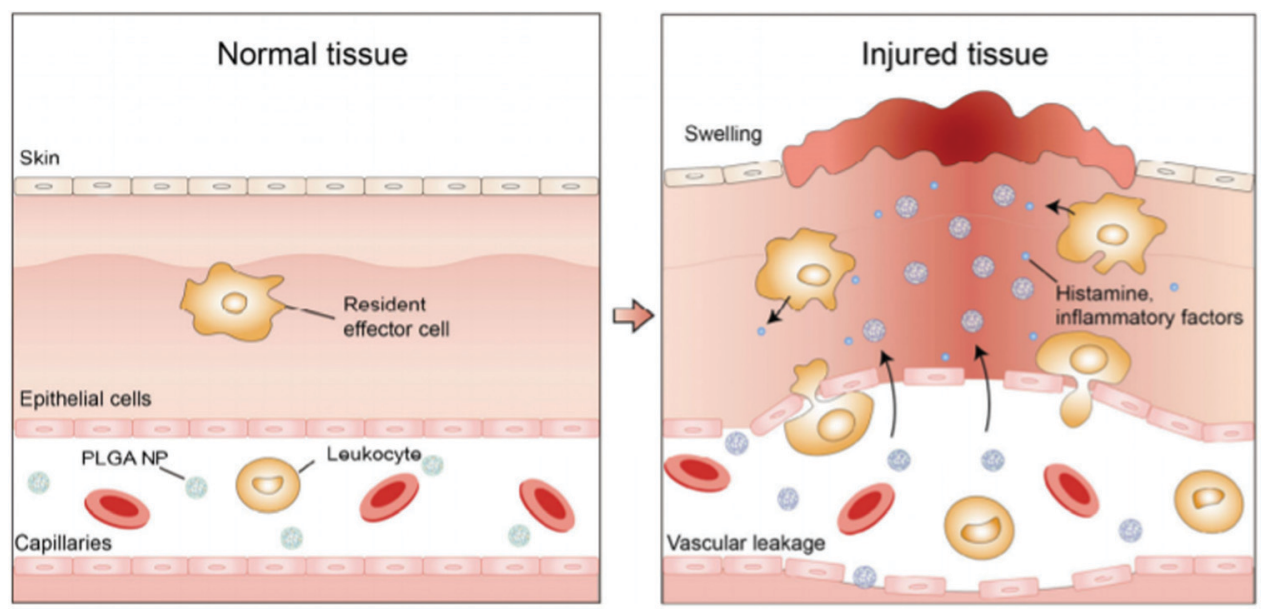

Fig. 2 The scheme of the EPR effect at the site of injury (reprinted with permission from Nano Research, 2020, 13, 564 ${ }^{34}$ ).

preserved to the maximum. Noteworthily, it has recently been discovered that the presence of plasma albumin will not worsen VILI, as long as the mechanical ventilation does not collapse the endogenous surfactant monolayer. ${ }^{1}$ Thus, the function of exogenous surfactant should not be compromised by albumin. Exogenous surfactant, when intravenously administered in nano-particle capsules, is expected to reach the targeted lung regions as intended via inflammation-induced high capillary permeability across a concentration gradient. Systemic absorption, metabolism, and excretion should remain minimal as compared with direct intravenous administration. Hence, with nanoparticle-driven more efficient administration, the exogenous surfactant should work towards improved oxygenation, reduced systemic inflammation, and lower VILI as shown in live animal models. $^{37}$

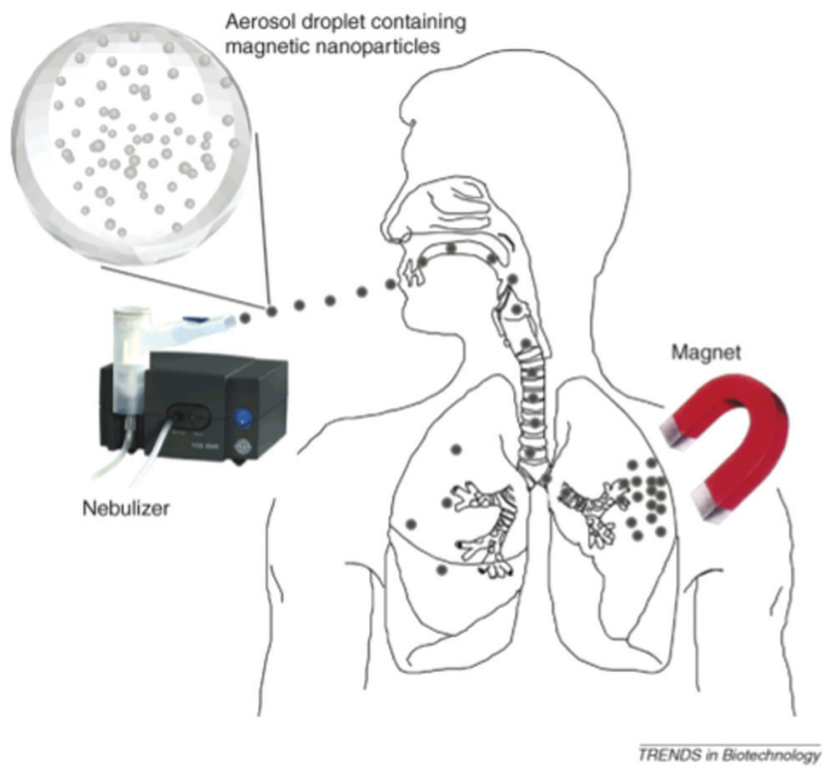

Fig. 3 Magnetic drug targeting of aerosol particles via the respiratory system (reprinted with permission from Trends Biotechnol, 2008, 26, $\left.59^{36}\right)$.
Clinical studies have provided a strong rationale for employing exogenous surfactants in COVID-19 patients. $^{23,38}$ In a retrospective case-control pilot study conducted by Portman and colleagues, ${ }^{23}$ seven COVID-19 PCR positive ARDS patients received liquid Curosurf (720 mg) in $150 \mathrm{ml}$ normal saline through a bronchoscope into second-generation bronchi. Patients showed no evidence of acute decompensation following surfactant installation into minor bronchi. Compared with 14 patients who received supportive care during the same period, surfactant administration reduced the 28 days mortality rate, although it was not significant due to the limited patient population. In addition, health care providers did not convert to COVID-19 positive. Kramer et al., present their experience with exogenous surfactant treatment in a patient with COVID19-related ARDS. The patient responded to the administered surfactant with improved oxygenation. ${ }^{39}$ Although there is a lack of a large number of patient samples to reflect how exogenous surfactant would benefit more patients, these studies show that surfactant therapy is feasible and safe for the treatment of COVID-19 induced respiratory failure, and potentially could be the reason for successful extubation and contribute to improving the clinical outcomes.

On the other hand, nanoparticle-based drug delivery systems offer multiple benefits in treating diseases, such as targeted drug delivery, protection of drugs from degradation, sustained release of specific amounts of drugs in a controlled manner, and multiple administration routes. Since 1995, more than 50 nanoparticles have received Food and Drug Administration (FDA) approval and more than 70 products are in clinical trials. ${ }^{40}$ These nanoparticle-based formulations are widely used for the clinical treatment of various diseases, such as cancer, diabetes, cardiovascular diseases, and rheumatoid arthritis. In the last few decades, nanoparticles have significantly boosted the development of RNA therapeutics, because nanoparticles can accommodate RNA molecules to increase their stability and enhance their intracellular release efficacy. ${ }^{41-45}$ The most recent example is that lipid nanoparticles are used as mRNA vehicles in the BioNTech/Pfizer's BNT162b2 and Moderna's mRNA-1273 vaccines. ${ }^{46}$ The successful 
record of nanoparticles in disease treatment and COVID-19 vaccine development, as well as the positive results of the clinical studies of surfactant therapy on COVID-19 patients, support future clinical trials that evaluate the efficacy of nanoparticle-mediated surfactant therapy for patients with COVID-19 ARDS.

\section{Conclusions}

In this perspective, we propose to employ nanoparticle-enabled surfactant therapy to provide therapeutic benefits for COVID19-associated ARDS to avoid the occurrence or reduce the amount of VILI. The success of nanoparticles in drug delivery and the current practice of using lipid nanoparticles as a vital component of the new Pfizer/BioNTech and Moderna mRNA COVID-19 vaccines make the proposed solution feasible in the following aspects. First, lipid nanoparticle platforms protect the mRNA sequence against being degraded and transport the mRNA effectively to the cytosol by endocytosis. ${ }^{47}$ Similarly, nanoparticles could protect the surfactant from being degraded or eliminated from the body and transport the surfactant to the intended lung regions that need surfactant most. Second, despite their clear advantages for drug delivery, certain components of nanoparticles have unwanted side effects. For example, the PEG component in the Pfizer/BioNTech and Moderna mRNA COVID-19 vaccines is believed to cause severe allergic responses, ${ }^{48}$ particularly for those who suffer from severe allergies. ${ }^{49}$ Therefore, if PEG is formulated in nanoparticles for surfactant therapy, the nanoparticle platform may cause allergic responses.

There are extra issues about translating engineering mechanisms into clinical practice that we must take into consideration when applying nanoparticle-driven surfactant therapy. For example, our proposed scheme includes an external magnetic field to guide the transport of nanoparticles. Those COVID-19 patients with a pacemaker or implantable defibrillators should be excluded. ${ }^{50}$ Also, ARDS caused by different mutations in COVID-19 may be different in pathophysiology, and targeting surfactant delivery is less likely to improve all COVID19-associated ARDS patients. Thus, the pharmacological and toxicological issues in nano-medicine for COIVD-19associated-ARDS are expected to diversify and corresponding clinical practices should be individualized, if possible. ${ }^{51}$

Collectively, COVID-19 could cause ARDS in severely attacked subjects, in which the endogenous lung surfactant system is compromised, leading to prolonged mechanical ventilation, worse VILI, and even death. The administration of nano-particle-mediated exogenous surfactants is a potential treatment option to reduce the severity of disease in COVID 19 patients. Case reports demonstrated surfactant therapy for COVID 19 treatment and improved clinical oxygenation. Motivated by addressing the present challenges in traditional surfactant administration and inspired by the success of nanomedicine in other disease treatments, we discussed the use of nanoparticles to administer exogenous replacement surfactants. By enveloping exogenous surfactants into nanoparticles, their efficacy can be potentially improved by targeted delivery to the diseased lung regions and preventing the surfactant complex from losing its surface-active properties during transportation. In addition, since it has less interference to other organs and tissues, the side effects of surfactants are expected to be reduced. We expect nano-particle-medicated surfactant therapy to deliver surfactant to intended lung regions, improve lung compliance and oxygenation, and eventually reduce mortality.

\section{Author contributions}

Y. W. and C. Z. conceived the presented idea. Y.W., X. L., Y. G., and C. Z. wrote the paper.

\section{Conflicts of interest}

The authors declare no conflict of interest.

\section{Acknowledgements}

C. Z. acknowledges the support of start-up package and the Level 1-Standard RGC Project/RGC Seed Project (GR14901) from The University of Alabama. C. Z. is a recipient of the National Institutes of Health Research Enhancement Award (R15GM139193).

\section{References}

1 A. B. Kharge, Y. Wu and C. E. Perlman, J. Appl. Physiol., 2014, 117, 440-451.

2 A. H. Attaway, R. G. Scheraga, A. Bhimraj, M. Biehl and U. Hatipoğlu, BMJ, 2021, 372, n436.

3 W.-J. Guan, Z.-Y. Ni, Y. Hu, W.-H. Liang, C.-Q. Ou, J.-X. He, L. Liu, H. Shan, C.-L. Lei, D. S. C. Hui, B. Du, L.-J. Li, G. Zeng, K.-Y. Yuen, R.-C. Chen, C.-L. Tang, T. Wang, P.-Y. Chen, J. Xiang, S.-Y. Li, J.-L. Wang, Z.-J. Liang, Y.-X. Peng, L. Wei, Y. Liu, Y.-H. Hu, P. Peng, J.-M. Wang, J.-Y. Liu, Z. Chen, G. Li, Z.-J. Zheng, S.-Q. Qiu, J. Luo, C.-J. Ye, S.-Y. Zhu and N.-S. Zhong, N. Engl. J. Med., 2020, 382, 1708-1720.

4 P. G. Gibson, L. Qin and S. H. Puah, Med. J. Aust., 2020, 213, 54-56.e51.

5 C. Wu, X. Chen, Y. Cai, J. A. Xia, X. Zhou, S. Xu, H. Huang, L. Zhang, X. Zhou, C. Du, Y. Zhang, J. Song, S. Wang, Y. Chao, Z. Yang, J. Xu, X. Zhou, D. Chen, W. Xiong, L. Xu, F. Zhou, J. Jiang, C. Bai, J. Zheng and Y. Song, JAMA Intern. Med., 2020, 180, 934-943.

6 A. C. Borczuk, S. P. Salvatore, S. V. Seshan, S. S. Patel, J. B. Bussel, M. Mostyka, S. Elsoukkary, B. He, C. D. Vecchio, F. Fortarezza, F. P. N. Pezzuto, Andrea Crisanti, M. E. Fowkes, C. H. Bryce, F. Calabrese and M. B. Beasley, Modern Pathology, 2020, 33, 2156-2168.

7 Z. Xu, L. Shi, Y. Wang, J. Zhang, L. Huang, C. Zhang, S. Liu, P. Zhao, H. Liu, L. Zhu, Y. Tai, C. Bai, T. Gao, J. Song, P. Xia, J. Dong, J. Zhao and F.-S. Wang, Lancet Respir. Med., 2020, 8, 420-422. 
8 L. Gattinoni, D. Chiumello, P. Caironi, M. Busana, F. Romitti, L. Brazzi and L. Camporota, Intensive Care Med., 2020, 46, 1099-1102.

9 J. J. Marini and L. Gattinoni, JAMA, J. Am. Med. Assoc., 2020, 323, 2329-2330.

10 L. Sang, X. Zheng, Z. Zhao, M. Zhong, L. Jiang, Y. Huang, X. Liu, Y. Li and D. Zhang, Front. Med., 2021, 7.

11 L. Gattinoni, S. Coppola, M. Cressoni, M. Busana, S. Rossi and D. Chiumello, Am. J. Respir. Crit. Care Med., 2020, 201, 1299-1300.

12 R. J. Mason, Eur. Respir. J., 2020, 55, 2000607.

13 L. Gattinoni, K. Meissner and J. J. Marini, Intensive Care Med., 2020, 46, 1438-1440.

14 S. Sardesai, M. Biniwale, F. Wertheimer, A. Garingo and R. Ramanathan, Pediatr. Res., 2017, 81, 240-248.

15 R. A. Polin and W. A. Carlo, Pediatrics, 2014, 133, 156-163.

16 J. Kesecioglu, R. Beale, T. E. Stewart, G. P. Findlay, J.-J. Rouby, L. Holzapfel, P. Bruins, E. J. Steenken, O. K. Jeppesen and B. Lachmann, Am. J. Respir. Crit. Care Med., 2009, 180, 989-994.

17 K. Raghavendran, D. Willson and R. H. Notter, Crit. Care Clin., 2011, 27, 525-559.

18 E. Herting, C. Härtel and W. Göpel, Arch. Dis. Child., 2019, 104, F655-F659.

19 D. Halpern, O. E. Jensen and J. B. Grotberg, J. Appl. Physiol., 1998, 85, 333-352.

20 J. A. Whitsett and T. Alenghat, Nat. Immunol., 2015, 16, 27-35.

21 X. M. Bustamante-Marin and L. E. Ostrowski, Cold Spring Harbor Perspect. Biol., 2017, 9, a028241.

22 J. B. Grotberg, M. Filoche, D. F. Willson, K. Raghavendran and R. H. Notter, Am. J. Respir. Crit. Care Med., 2017, 195, 538-540.

23 S. Piva, R. M. DiBlasi, A. E. Slee, A. H. Jobe, A. M. Roccaro, M. Filippini, N. Latronico, M. Bertoni, J. C. Marshall and M. A. Portman, Respir. Res., 2021, 22, 20.

24 N. El-Gendy, A. Kaviratna, C. Berkland and P. Dhar, Ther. Delivery, 2013, 4, 951-980.

25 M. Filoche, C.-F. Tai and J. B. Grotberg, Proc. Natl. Acad. Sci. U. S. A., 2015, 112, 9287-9292.

26 M. H. Chua, W. Cheng, S. S. Goh, J. Kong, B. Li, J. Y. C. Lim, L. Mao, S. Wang, K. Xue, L. Yang, E. Ye, K. Zhang, W. C. D. Cheong, B. H. Tan, Z. Li, B. H. Tan and X. J. Loh, Research, 2020, 2020, 7286735.

27 Z. Tang, N. Kong, X. Zhang, Y. Liu, P. Hu, S. Mou, P. Liljeström, J. Shi, W. Tan, J. S. Kim, Y. Cao, R. Langer, K. W. Leong, O. C. Farokhzad and W. Tao, Nat. Rev. Mater., 2020, 5, 847-860.

28 R. Torrente-Rodríguez, H. Lukas, J. Tu, J. Min, Y. Yang, C. Xu, H. Rossiter and W. Gao, Matter, 2020, 3.

29 L. Schoenmaker, D. Witzigmann, J. A. Kulkarni, R. Verbeke, G. Kersten, W. Jiskoot and D. J. A. Crommelin, Int. J. Pharm., 2021, 601, 120586.
30 M. Mehta, P. Prasher, M. Sharma, M. D. Shastri, N. Khurana, M. Vyas, H. Dureja, G. Gupta, K. Anand, S. Satija, D. K. Chellappan and K. Dua, Med. Hypotheses, 2020, 144, 110254.

31 Z. Tang, X. Zhang, Y. Shu, M. Guo, H. Zhang and W. Tao, Nano Today, 2021, 36, 101019.

32 A. Wicki, D. Witzigmann, V. Balasubramanian and J. Huwyler, J. Controlled Release, 2015, 200, 138-157.

33 S. H. van Rijt, T. Bein and S. Meiners, Eur. Respir. J., 2014, 44, 765-774.

34 Y. Liu, D. Sun, Q. Fan, Q. Ma, Z. Dong, W. Tao, H. Tao, Z. Liu and C. Wang, Nano Res., 2020, 13, 564-569.

35 P. Dames, B. Gleich, A. Flemmer, K. Hajek, N. Seidl, F. Wiekhorst, D. Eberbeck, I. Bittmann, C. Bergemann, T. Weyh, L. Trahms, J. Rosenecker and C. Rudolph, Nat. Nanotechnol., 2007, 2, 495-499.

36 C. Plank, Trends Biotechnol., 2008, 26, 59-63.

37 Y. Wu, T. L. Nguyen and C. E. Perlman, J. Appl. Physiol., 2021, 130, 1305-1316.

38 A. C. Koumbourlis and E. K. Motoyama, Am. J. Respir. Crit. Care Med., 2020, 202, 624-626.

39 M. Heching, S. Lev, D. Shitenberg, D. Dicker and M. R. Kramer, Chest, 2021, DOI: 10.1016/j.chest.2021.01.028.

40 C. L. Ventola, J. Clin. Pharm. Ther., 2017, 42, 742-755.

41 J. C. Kaczmarek, P. S. Kowalski and D. G. Anderson, Genome Med., 2017, 9, 60.

42 X. Xu, J. Wu, Y. Liu, P. E. Saw, W. Tao, M. Yu, H. Zope, M. Si, A. Victorious, J. Rasmussen, D. Ayyash, O. C. Farokhzad and J. Shi, ACS Nano, 2017, 11, 2618-2627.

43 M. A. Islam, Y. Xu, W. Tao, J. M. Ubellacker, M. Lim, D. Aum, G. Y. Lee, K. Zhou, H. Zope, M. Yu, W. Cao, J. T. Ostwald, M. Dinarvand, M. Mahmoudi, R. Langer, P. W. Kantoff, O. C. Farokhzad, B. R. Zetter and J. Shi, Nat. Biomed. Eng., 2018, 2, 850-864.

44 N. Kong, W. Tao, X. Ling, J. Wang, Y. Xiao, S. Shi, X. Ji, A. Shajii, S. T. Gan, N. Y. Kim, D. G. Duda, T. Xie, O. C. Farokhzad and J. Shi, Sci. Transl. Med., 2019, 11, eaaw1565.

45 W. Tao, A. Yurdagul, N. Kong, W. Li, X. Wang, A. C. Doran, C. Feng, J. Wang, M. A. Islam, O. C. Farokhzad, I. Tabas and J. Shi, Sci. Transl. Med., 2020, 12, eaay1063.

46 Nat. Rev. Mater., 2021, 6, 99.

47 N. Pardi, M. J. Hogan, F. W. Porter and D. Weissman, Nat. Rev. Drug Discovery, 2018, 17, 261-279.

48 K. G. Blumenthal, L. B. Robinson, C. A. Camargo, Jr, E. S. Shenoy, A. Banerji, A. B. Landman and P. Wickner, JAMA Intern. Med., 2021, 325, 1562-1565.

49 J. A. Bellanti and R. A. Settipane, Allergy Asthma Proc., 2020, 41, 229-231.

50 R. Beinart and S. Nazarian, Circulation, 2013, 128, 2799-2809.

51 J. S. Brenner, Ann. Am. Thorac. Soc., 2017, 14, 561-564. 